\title{
Delivering bad news: a vital skill for professionals involved in breast cancer care
}

\author{
M. N. Linver ${ }^{a, b}$ \\ ${ }^{a} X$-ray Associates of New Mexico, P.C., Albuquerque, NM, USA; ${ }^{b}$ Clinical Professor of Radiology, \\ University of New Mexico School of Medicine, Albuquerque, NM, USA.
}

\begin{abstract}
Delivering bad news to patients being evaluated for and diagnosed with breast cancer requires a unique blend of medical knowledge, compassion, and sensitivity. Although many of the techniques involved are intuitive, one can enhance these skills by incorporating a few simple concepts into the patient encounter. By combining these with an understanding of appropriate responses to possible patient reactions to the news, one can create a more effective groundwork for the patient's recovery on both physical and emotional levels.
\end{abstract}

Keywords: Breast cancer; Cancer treatment; Patient communication; Physician-patient communication

\section{Introduction}

Those of us who are involved in the diagnosis or treatment of breast cancer face many difficult tasks in our daily interactions with patients. Among the most difficult is one for which we, as physicians, are least prepared: delivering the news that the patient has breast cancer. This is especially true for breast imagers, who have taken on an increasing clinical role in this evolving subspecialty area of radiology, although the task of delivering such news falls upon almost all physicians to varying degrees. As a guide for those involved, a review of some of the important skills and issues related to delivery of bad news is offered here. What follows represents the lessons learned by the author from his 22 years of experience as a breast imager [1], infused with additional information adapted from Harvey et al. in their article, 'Breaking bad news: a primer for radiologists in breast imaging' [2],

Correspondence to: Michael N. Linver, MD, FACR, 6504 Avenida La Cuchilla, NW, Albuquerque, NM 87107, USA. E-mail: mammomike@ aol.com; Tel: 505-998-1912; Fax: 505-998-3100

Received: $21 / 10 / 08$

Accepted: 16/01/09

First published online 23/06/09

BCO/806/2008/FO and from Buckman in his book, 'How to break bad news: a guide for health care professionals' [3].

\section{Delivering bad news: why the need to do it well?}

Correctly diagnosing and treating breast cancer remains our primary focus. However, we are dealing with human beings, and must respond to their emotional needs as well. They desperately need our support in order to allay their fears and give them hope at this devastating time in their lives. By delivering this news adeptly, and by being sensitive to the patients' responses, we can set the tone for their successful journey back to stability and good health.

\section{The bad news bearers: how to do it?}

The first step is to put one's self in the patient's place. Such empathy is a valuable barometer in helping guide one's interaction with the patient. One should approach the patient in a comfortable setting, preferably a quiet room without interruptions. In order to reduce the patient's sense of helplessness in this setting, one should be at eye level 
with the patient. Physical contact is critical to establishing a bond of caring and concern: shaking the patient's hand or even gently touching her arm should be included at the outset of the interchange.

In beginning the discussion, one should avoid medical jargon as much as possible. This policy can be varied to some extent as one gauges the patient's level of understanding. It is wise to ask the patient to bring with her a family member or friend. The patient will often hear almost nothing that is said after the word 'cancer' is uttered. Therefore, another 'active listener' can act as a valuable source of information to the patient after the session is completed.

As the discussion ensues, it is best not to use the word 'cancer' very often: the word is still synonymous with death to many patients. In addition, it is important to dwell on the most positive aspect of the situation. For instance, one should emphasize the small size of the tumor or its low grade, if these features are present. By relating these aspects to a higher likelihood of 'cure', one offers the patient great comfort and hope during this difficult time, and provides the emotional sustenance she will need to survive the painful road ahead.

Once finished delivering the news, one should allow a period of silence so that the patient might collect her thoughts, and then ask her if she has any thoughts or concerns. After addressing these, one must then be prepared to give the patient very specific instructions as to her next steps. It is best to have already established communication with the breast surgeon, the medical oncologist, the radiation oncologist, and the rest of the team to be involved in the patient's treatment, and to have already scheduled her for the appropriate appointment(s). At this juncture, one might remind the patient to write down any questions she may have for these specialists, and to be prepared to present them at the time of her appointment(s). One should suggest that a family member or friend accompany her to be an 'active listener' at these appointments as well.

The patient will now require not only structure to her care, but 'navigation' through the system, as she will likely be in no position to do so on her own. Therefore, one should arrange to have a resource person available to offer continued emotional and physical support, provide information, help with insurance matters, and possibly even escort her to her next appointment. In our breast imaging center, we have a full-time nurse navigator whose primary role is to provide such support for the newly diagnosed breast cancer patient. This individual provides a physical link between the breast imaging physician and the next specialist along the patient's chain of care, and between the patient and her entire breast care 'team'.

Again, it is important to emphasize that the patient often hears almost nothing the physician explains to her during the encounter. Thus, one must also provide a means for her to communicate after she has digested the news more completely, and has collected her thoughts. Accordingly, one should provide the patient with a business card listing a phone number or email address to 'keep the door open' and maintain that critical lifeline for the patient.

\section{Dealing with patient reactions}

As in all stressful situations, patients cope with bad news in highly variable ways. These include shock and disbelief, denial, information gathering, fear and anxiety, anger, blame, guilt, and humor, among others. As much as possible, one should anticipate and formulate a response to each of these. The most common reaction is shock and disbelief, especially if the patient's cancer was discovered on a screening mammogram, with no clinical findings to act as warning signs to the patient. Sometimes the breast imager can obviate this reaction by giving a strong warning shot when the patient was first called back for evaluation of the abnormal finding at screening, even before any biopsy was performed. The elements of surprise and shock may then be partly mitigated.

If the patient responds with prolonged silence after being given the bad news, this may be a manifestation of shock. In this situation, it is best to let the silence hang while the patient processes the information. She may thereby be able to overcome her initial shock and respond appropriately.

Another common reaction is denial. If confronted by such a reaction, one might offer an empathetic response such as 'I know this information must be hard to accept because it is so unexpected', followed immediately by a discussion of a treatment plan. This approach may break through the barrier of denial, but often the tincture of time is still required before complete success is achievable.

A further reaction seen as a form of displaced grief is known as information gathering. This can be a very empowering coping mechanism, as it allows the patient some sense of control. Often, this will manifest in the patient's asking for a second opinion. It is then best to allow her to do so. Eventually, the displaced grief associated with this response will usually be admitted, allowing appropriate emotional processing to occur.

Yet another common reaction is fear and anxiety. Here, a response such as 'What is it that worries 
you most?' will often allow the patient to communicate her concerns, thereby overcoming her initial reaction.

Patients may manifest anger or an aggressive response to bad news due to underlying fear, and may lash out directly at the physician. Here, one should never respond with aggression in turn, no matter how provoking the patient has been. Instead, one should attempt to find the underlying cause of the anger, and address it in a calm and non-threatening manner. Such an approach will usually allow the patient's anger to slowly dissipate.

Often, the patient's anger is shifted to blame a particular individual. Again, a discussion of the patient's feelings will usually resolve the tension. Here, it is critical not to validate the blame being placed by the patient, especially toward other health care providers. Instead, providing appropriate empathy is the best response. The patient may also blame herself for her situation rather than others. Again, an empathetic response will enable the patient to discuss her sense of guilt, and hopefully resolve it.

Crying is another frequent reaction for which one should always be prepared. Watching the patient's eyes and providing tissues at the first sign of tearing are always helpful. One should be supportive as the patient calms down, and should offer her the support of a family member or friend before leaving the room. In this way, the patient will not undergo any sense of abandonment.

It is important for the physician to recognize when his or her own stress level is so high that warm and empathetic feelings toward the patient's reactions have been compromised, and the physician begins to experience anger. If and when this occurs, it is usually best for a colleague to take over the patient's care. The appearance of physician's anger can completely destroy the bond of trust with the patient, and can be devastating to both.

\section{Summary}

The physician who delivers bad news to the breast cancer patient must be prepared to deal with the emotional needs of the patient as well as her physical needs. One should be able to perform this task with a sense of genuine empathy and caring that provide the support needed by the patient at this defining moment. At the same time, the physician should remember that the patient may hear almost nothing said to her during the encounter, and must be given the opportunity to seek out the physician with future questions, if necessary. The physician must likewise be prepared to deal with a variety of possible reactions manifested by the patient, and guide the patient through this gamut of emotional turmoil to its successful resolution. By doing so, the physician and patient can establish a special bond that transcends the pure science and embraces the real art of medicine. It is only then that the healing process can truly begin.

\section{References}

1. Linver MN. Connecting with your patients. Am Coll Radiol Bulletin 2008; 63(2): 24-25.

2. Harvey JA, Cohen MA, Brenin DR, et al. Breaking bad news: a primer for radiologists in breast imaging. $J \mathrm{Am}$ Coll Radiol 2007; 4: 800-808.

3. Buckman R. How to Break Bad News: A Guide for Health Care Professionals. Baltimore, MD: Johns Hopkins University Press, 1992. 